\section{Vietnam Journal of Agricultural Sciences}

\title{
Expression of Zinc Finger Protein Zat12 from Arabidopsis thaliana in Escherichia coli
}

\author{
Le Thi Tuyet Cham, Vu Ngoc Thang, Tran Anh Tuan \& Vu Thi \\ Thuy Hang
}

Faculty of Agronomy, Vietnam National University of Agriculture, Hanoi 131000, Vietnam

\begin{abstract}
The $\mathrm{C} 2 \mathrm{H} 2$ zinc finger protein ZAT12 has been classified as a plant core abiotic stress response gene in the early response to multiple stresses. ZAT12 links the iron deficiency and oxidative stress responses through the direct interaction with/and negative regulation of a central regulator - FIT. For further research on the regulation of the ZAT12 protein in planta, a huge quantity of ZAT12 proteins is required to inject into mice for the generation of ZAT12 antiserum. In this study, the gene encoding the ZAT12 protein from Arabidopsis thaliana was cloned into the expression vector - pETBlue- 2 and then overexpressed in E. coli T7. A high expression level was indicated by SDS-PAGE. Immunoblot demonstrated successful expression using a bacterial expression system.
\end{abstract}

\section{Keywords}

ZAT12, zinc finger protein, ZAT12 expression

\section{Introduction}

The zinc finger of Arabidopsis thaliana 12 (ZAT12), a member of the $\mathrm{C} 2 \mathrm{H} 2$-type plant-specific zinc $(\mathrm{Zn})$ finger transcription factor family (Englbrecht et al., 2004; Ciftci-Yilmaz and Mittler, 2008; Miller et al., 2008; Kiełbowicz-Matuk, 2012), contains an EAR motif and is thought to function as a repressor of gene expression (Kagale et al., 2010). ZAT12 was identified among genes induced by light (Iida et al., 2000; Davletova et al., 2005b), low temperatures (Fowler \& Thomashow, 2002; Kreps et al., 2002; Vogel et al., 2005), wounding (Cheong et al., 2002), osmotic and salinity stress (Kreps et al., 2002), and oxidative stress (Rizhsky et al., 2004; Davletova et al., 2005b; Vanderauwera et al., 2005). ZAT12 is also the direct target of many transcriptional regulators such as EIN3, bZIP29, AtUSB1, DRM2, At3g03170, and LEA18 (Peng et al., 2014; Ben Daniel et al., 2016). However, the role of the ZAT12 protein in the abiotic stress signaling network has not been fully elucidated.

Recently, ZAT12 was identified in an attempt to find a link between iron deficiency responses and oxidative stress. In conditions 
where iron is poorly available, plants are able to induce a set of genes that enhance the efficiency of its uptake by the roots. A central regulator of this response in the model plant Arabidopsis (Arabidopsis thaliana) is the basic helix-loophelix transcription factor FIT whose activity is tightly regulated. Using a yeast two-hybrid assay and BiFC, the EAR motif was demonstrated to be necessary for the interaction between FIT and ZAT12 (Le et al., 2016). The expression of the FIT gene was upregulated in zat12 loss-offunction plants. In addition, these plants accumulated a higher amount of iron compared to the wild type. ZAT12-GFP fluorescence was detected in Arabidopsis roots, where it could be observed in the nuclei. This protein was elevated upon iron deficiency, $\mathrm{H}_{2} \mathrm{O}_{2}$, and MG132 treatments. ZAT12 is considered as a link between iron deficiency and oxidative stress responses through direct interaction with and negative regulation of FIT. These functions of the ZAT12 protein were only demonstrated using a ZAT12-GFP protein with a GFP antibody in immunoblot experiments (Le et al., 2016). To verify this native ZAT12 function, i.e to monitor $\mathrm{Fe}$ dependent expression and the regulation of ZAT12 protein in planta, it is necessary to generate an anti ZAT12 antibody. To do this, we have conducted a series of experiments including the cloning, transformation, heterologous expression, and purification of the recombinant protein. In this study, the ZAT12 recombinant protein was successfully expressed in E. coli.

\section{Materials and Methods}

Strains, expression vector, and culture conditions for cloning

E. coli Nova Blue cells were used as hosts for transformation. E. coli strains carrying the pETBlue-2 vector (Figure 1) were grown in Luria-Bertani medium supplemented with $100 \mu \mathrm{g}$ of tetracycline $/ \mathrm{mL}$ when required at $37^{\circ} \mathrm{C}$. The DNA fragments for subcloning were isolated from agarose gels and cloned into the pETBlue2 vector using a Perfectly Blunt ${ }^{\circledR}$ Cloning Kit. (Novagen, Germany). Tuner (DE3) pLacI E. coli host strains that are compatible with the pET expression systems from Novagen were used for the expression of the ZAT12 protein. Other standard microbial and recombinant techniques used throughout this work were as described by Sambrook et al. (2001).

\section{Generation of the ZAT12-His gene constructs using PCR}

The coding sequence of ZAT12 from Arabidopsis thaliana was amplified by PCR using the primer combination 5' ZAT12_PET (5'-ATGGTTGCGATATCGGAGATCAA - 3 ') (Figure 2) and ZAT12_CT His 3' (5'TCAAGAGGCCATACCGTGATGATGATGA TGATGAGAACCACGATAAACTGTTCT

TCCAAGCTCCA -3') (Le et al., 2016). After checking the size of these fragments by agarose electrophoresis, the ZAT12-His fragments were purified and cloned into the EcoRV cloning site of the pETBlue-2 vector using the Perfectly Blunt ${ }^{\circledR}$ Cloning Kit.

\section{Transformation}

The recombinant plasmid was transformed into NovaBlue Singles ${ }^{\mathrm{TM}}$ Competent Cells (Novagen, USA). Identification of successful cloning events was performed by blue/white screening. The insertion of ZAT12-His into the pETBlue-2 vector leads to the disruption of the expression of the lac $Z \alpha$-peptide, and thereby produces white colonies on plates supplemented with $\mathrm{X}$-gal and IPTG (Isopropyl- $\beta$-D_thiogalactoside from Roth, Karlsruhe, Germany). Inversely, colonies containing the vector without insertion turn blue. After the initial selection of positive colonies, colony PCR and sequencing were performed for verification of positive recombinant plasmids. After that, the recombinant plasmid was transformed into Tuner (DE3) pLacI cells and the recombinant protein induction was performed according to the manufacturer's instructions (Novagen, USA).

\section{Protein electrophoresis and Immuno Blot}

Sodium dodecyl sulfate-polyacrylamide gel electrophoresis (SDS-PAGE) was performed to determine the molecular mass of the protein using 10\% gels (Laemmli et al., 1970). 


\begin{tabular}{|c|c|}
\hline lacoperator & $3606-3625$ \\
\hline T7 promoter & $1-17$ \\
\hline lacoperator & $22-42$ \\
\hline T7 transcription start & 18 \\
\hline $\begin{array}{l}\text { multiple cloning region } \\
\text { (Ncol-PacI) }\end{array}$ & $276-467$ \\
\hline His•Tag" coding sequence & $437-454$ \\
\hline HSV - Tag $^{\circ}$ coding sequence & $395-430$ \\
\hline lacZstart codon & 491 \\
\hline lac $Z \alpha$-peptide ORF & $57-491$ \\
\hline E coli promoter & $541-569$ \\
\hline $\mathrm{fl}$ origin & $1096-1551$ \\
\hline bla coding sequence & $1669-2526$ \\
\hline pUC origin & 3206 \\
\hline
\end{tabular}

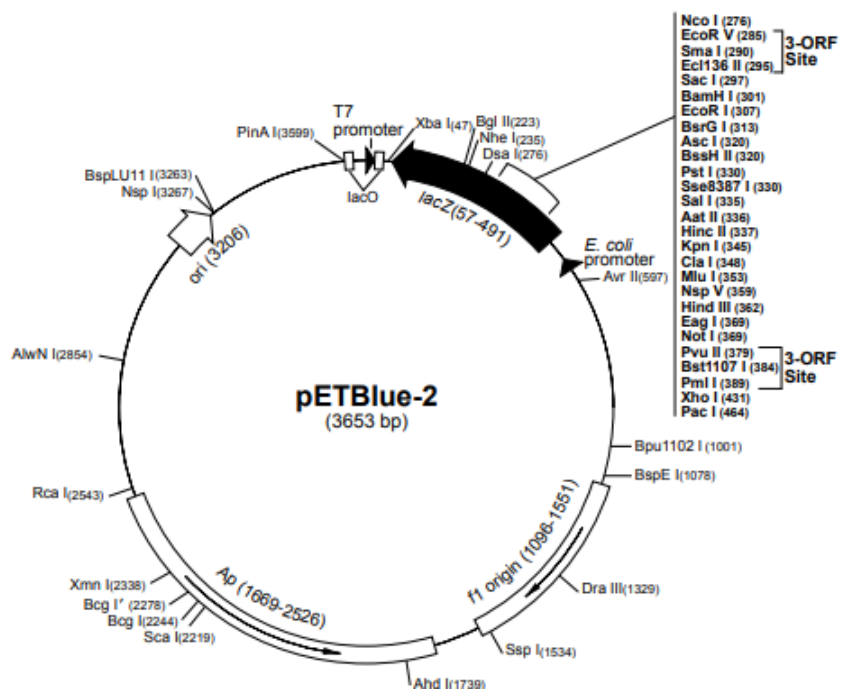

Figure 1. Map of the pETBlue-2-vector. Overview of the $p E T B l u e-2-v e c t o r$ map with multiple cloning sites (Novagen, Gernmany)

\section{5' ZAT12 PET}

Figure 2. Schematic view of the amplified fragment for cloning and colony PCR. A. The ZAT12 full DNA sequence was amplified using the 5'ZAT12_PET and ZAT12_CT His 3' primers. The 6x His Tag was added to the reverse primer. The size of the amplified fragment indicated the length of the ZAT12 full DNA with $6 x$ His sequence. $B$. The size of the colony PCR product was amplified using the 5' ZAT12_PET and pETBlue Down primers (from Novagen) after transformation.

The bacterial extract containing the recombinant ZAT12 fusion protein was loaded on a preparatory gel and blotted to a nitrocellulose membrane. After Ponceau $S$ staining, the membrane region containing the ZAT12 antigen was cut off as a strip. The membrane was blocked for 1 hour at room temperature with $1 \%$ bovine serum albumin (BSA) dissolved in PBS-T and subsequently probed with crude mouse antiserum at $4^{\circ} \mathrm{C}$ overnight. The unbound fraction was collected into a new tube. The membrane was washed 3 times with PBS-T and the bound antibodies were eluted two times with elution buffer $(0.1 \mathrm{M}$ glycine- $\mathrm{HCl} \mathrm{pH} 2.7,0.5 \mathrm{M} \mathrm{NaCl})$. The eluted antibody fractions were immediately neutralized by adding $1 / 10$ volume of neutralization buffer (1 M Tris- $\mathrm{HCl} \mathrm{pH} 8.0,1.5 \mathrm{M} \mathrm{NaCl}, 1 \mathrm{mM}$
EDTA, 0.5\% NaN3) and BSA was added at a 1 $\mathrm{mg} \mathrm{mL}^{-1}$ final concentration (Novagen, according to manufacturer's manual).

For the detection of the ZAT12-His protein, His antibodies and freshly purified undiluted antiZAT12 mouse antiserum were applied. These primary antibodies were detected with anti-mouse IgG conjugated with horseradish peroxidase (1:8000 dilution, Sigma-Aldrich, USA).

The ZAT12-His protein was detected by incubation with His rat antibody (1:1000 Roche, Germany) and a secondary antibody anti-rat IgG (whole molecule)-horse radish peroxidase conjugate (1:10000, Sigma-Aldrich, USA). Detection signals were developed using an enhanced chemiluminescence detection kit 
(Biorad, USA) according to the manufacturer's protocol.

\section{ZAT12 antibody preparation}

Based on the predicted antigenic propensity scores, a peptide corresponding to the $\mathrm{N}$-terminal of ZAT12 was chemically synthesized and conjugated with KLH (Bio Trend) and later injected into mice to obtain a polyclonal antiserum (this work was conducted by Prof. U. Müller, Zoology Department, Saarland University). In this study, the antiserum was tested for its specificity by detecting bacteria positively expressing ZAT12.

\section{Results}

Plasmid construction and confirmation of cloned recombinant ZAT12 plasmid

To generate the recombinant plasmid, the full-length DNA sequence of ZAT12 was specifically amplified with the ZAT12-PET and ZAT12-CT-His primers, which produced an expected band of 538bp. Upon successful ligation and transformation, the obtained colonies were numbered and a colony PCR was performed to check the positive clones for the presence of the recombinant plasmid. In addition, sequencing of the selected recombinant plasmid was performed to confirm the proper orientation of the insert by ligation (Figure 3).

Resultant colonies were tested for the presence of the recombinant plasmid by colony PCR, and colonies were numbered as $1,2,3, \ldots, 10$. If the insert was in the correct orientation, the expected size of the PCR product for ZAT12 with the primer combination (ZAT12 5' and pETBlueDOWN, see Figure 2) was approximately 800bp (538bp of ZAT12 full plus 232bp from the pET Blue2 vector). Colony no. 8 of ZAT12 gave a PCR product at the expected size.

\section{Expression of ZAT12 protein}

After verifying the sequence, the plasmid was transformed into BL21 (DE3) cells of E. coli for expression. SDS-PAGE analysis detected the expected products from BL21 (DE3) cells induced with IPTG. The results are shown in Figure 4.

After colony PCR confirmation, the selected recombinant plasmid was transformed into the Tuner ${ }^{\mathrm{TM}}$ DE3 expression cells. Upon successful expression of the recombinant $18 \mathrm{kDa}$ ZAT12His fusion protein at a small scale level, a large scale expression of ZAT12 protein was performed.
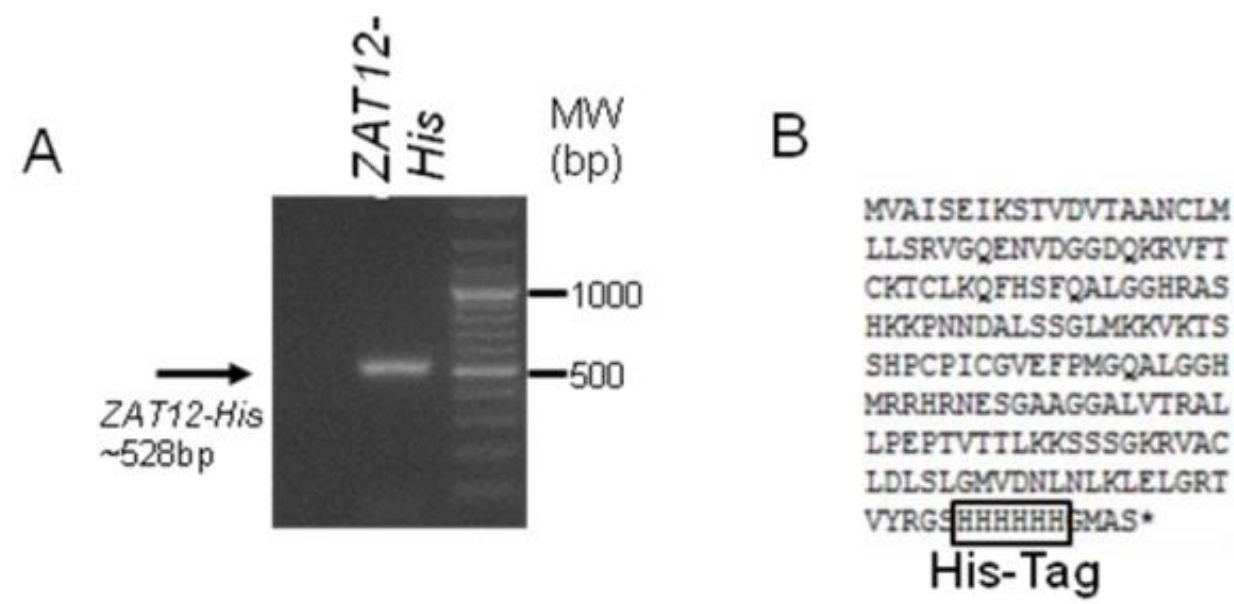

Figure 3. Preparation of the ZAT12 encoding gene. A. The DNA fragment of ZAT12-His using PCR. B. Amino acid changes at the 5'-ends of ZAT12 introduced via PCR. C. Colony-PCR of ZAT12-His colonies was performed on 10 colonies. Resultant colonies were numbered as $1,2,3, \ldots, 10$. Asterisks $\left(^{*}\right)$ indicate the $\sim 800 \mathrm{bp}$ size ZAT12 fusion DNA band (from $538 \mathrm{bp}$ of ZAT12 full plus $232 \mathrm{bp}$ from the pET Blue2 vector). The only colony no. 8 gave a PCR product at the expected size, Figure 1A. M=ladder. Colony no. 8 was a positive colony. 


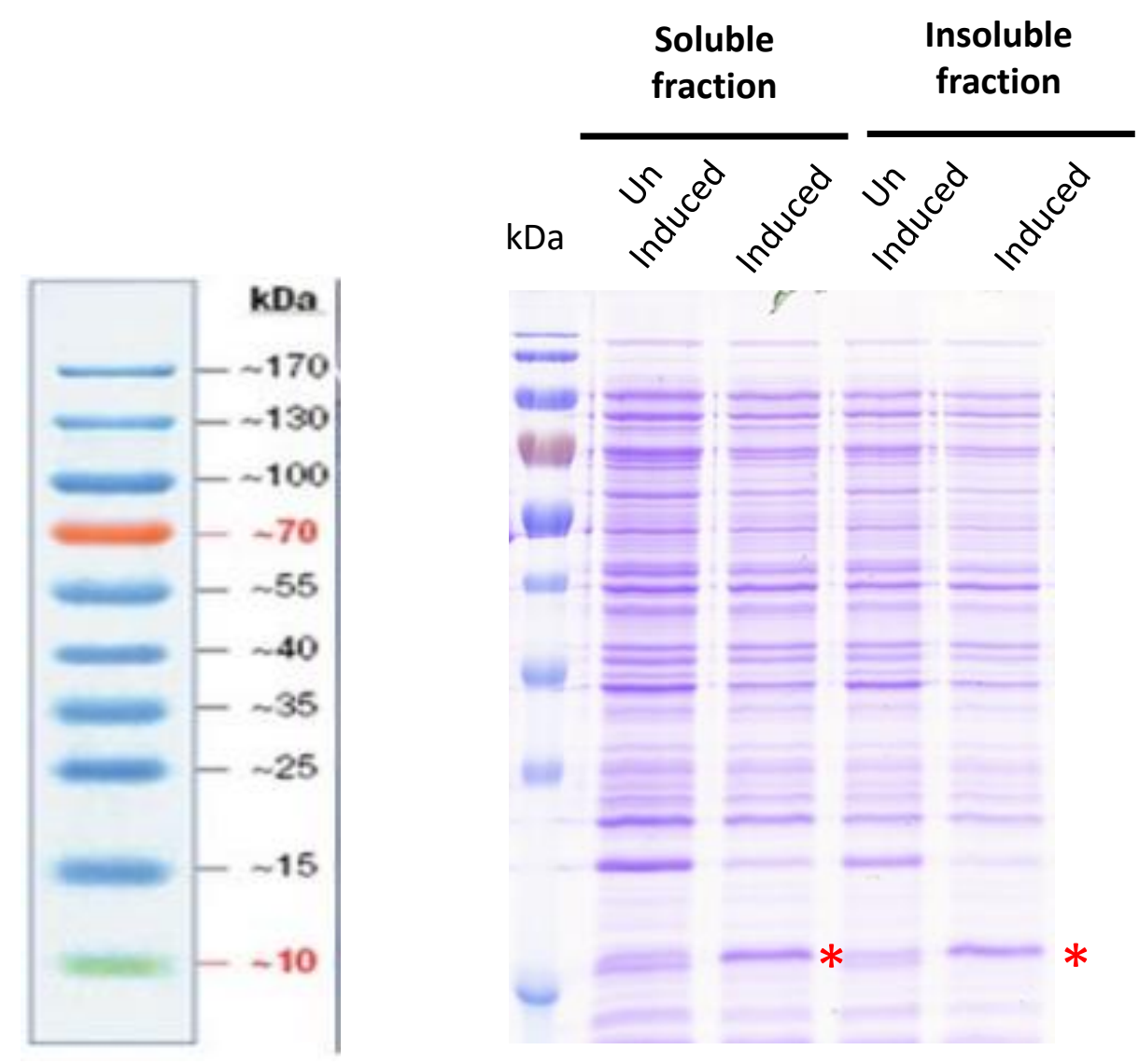

Figure 4. SDS-PAGE analysis of the heterologously expressed recombinant ZAT12 fusion protein in E. coli. ZAT12 fusion protein expression in Tuner ${ }^{T M}$ DE3 cells accumulated as insoluble proteins (inclusion bodies) and soluble fraction. Asterisks $\left({ }^{*}\right)$ indicate the position of $\sim 18 \mathrm{kDa}$ ZAT12 fusion protein band (from ZAT12 full gene plus 6 His tags).

\section{Specificity test of the ZAT12 protein}

The heterologously expressed recombinant ZAT12 was purified for affinity and injected into mice. The immunization of mice and collection of antiserum were kindly performed by Prof. Uli Müller and Iris Fuchs, Department of Zoology, Saarland University. The obtained antiserum was checked for its specificity on E. coli expressing the ZAT12 fusion protein (Figure 5). We detected a single band on western blot that matched to the specifically expressed and desired ZAT12 protein so that we were able to conclude that the ZAT12 protein was expressed successfully in E. coli.

\section{Discussion}

The $\mathrm{C} 2 \mathrm{H} 2$-type plant-specific zinc finger transcription factor family was defined by the presence of a conserved zinc finger domain, in which two cysteines and two histidines coordinate with a single zinc atom to form a finger construct consisting of a short beta-hairpin and an alpha helix. At5g59820 was identified among the genes belonging to this family and named ZINC FINGER OF ARABIDOPSIS THALIANA12 (ZAT12). Structural analysis of the ZAT12 protein showed that it constituted of 162 amino acids divided into two C2H2-type zinc finger domains with a 22-amino acid interfinger region, and a LDLSL core sequence of the EAR motif localized at the $\mathrm{C}$ terminus from amino acid 143 (Meissner \& Michael, 1997; Englbrecht et al., 2004; Kagale et al., 2010). In plants, the ethylene-responsive element binding factor associated with the Amphiphilic Repression (EAR) motif is a transcriptional regulatory motif found as an active repressor in members of the ERF, $\mathrm{C} 2 \mathrm{H} 2$, and AUX/IAA families, among others (Kagale et al., 2010). 

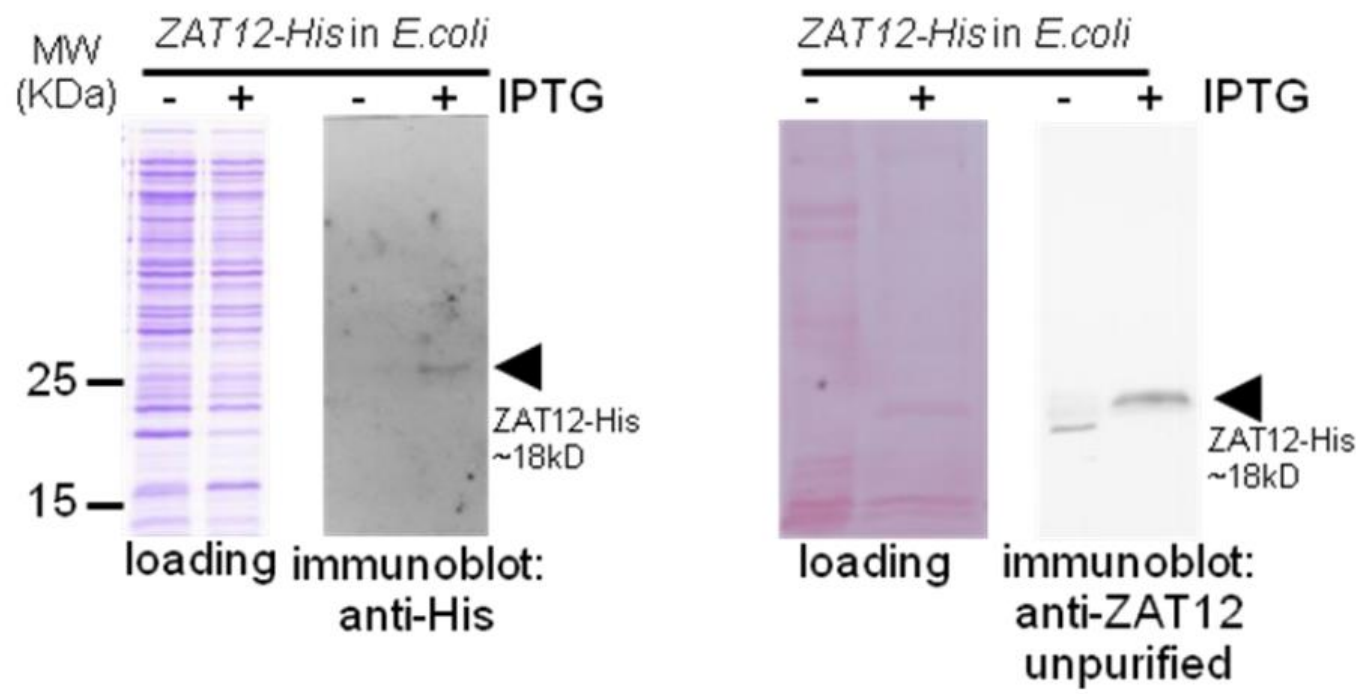

Figure 5. Specificity of the ZAT12 protein. A. The last lane from the right-hand side was loaded with ZAT12 induced bacterial culture and probed/incubated with anti-His antibodies (A), and with anti-ZAT12 unpurified antiserum (B). The triangle (1) indicates the position of the $\sim 18 \mathrm{kDa}$ ZAT12 fusion protein band (from ZAT12 full gene plus 6 His tags).

At5g59820 was identified to be a homolog of the Indica rice ZOS3-22 (Os03g0820400, LOC_Os03g6057, ZFP37). ZOS3-22 expression was also up-regulated in seedlings with a $\mathrm{H}_{2} \mathrm{O}_{2}$ treatment and in the shoots of plants exposed to Fe deficiency or combined Fe and P deficiency (de Abreu Neto \& Frei, 2015).

The coding sequence of ZAT12 from Arabidopsis thaliana was amplified using touchdown PCR. The annealing temperature of $58^{\circ} \mathrm{C}$ was suitable for amplification of the ZAT12 gene. Determination of the optimum annealing temperature for PCR is very important because total genomic DNA extracted from Arabidopsis thaliana was used as a template for ZAT12 amplified PCR (Rychlik et al., 1990). The fragment was a band with a size of 538bp, similar to the expected ZAT12 fragment size suggesting successful amplification of Zat12 from Arabidopsis thaliana genomic DNA using ZAT12_PET and ZAT12_CT His primers at the annealing temperature of $58^{\circ} \mathrm{C}$.

The transformed Nova Blue bacterial cells were selected using $100 \mu \mathrm{g} \mathrm{mL}^{-1}$ tetracycline due to the presence of the tetracycline resistance gene in pETBlue-2. Colony PCR on the randomly selected bacterial colonies was conducted using the primers ZAT12 5' and pETBlue DOWN which binds to the vector downstream of the cloning site to identify transformed bacteria that carry recombinant pETBlue-2 with Zat12 inserted at the desired orientation. Also, the amplified fragment was approximately $800 \mathrm{bp}$, consisting of 538bp of ZAT12 full and 272bp from the pET Blue2 vector. Lane 8 displayed a band with a size of approximately $800 \mathrm{bp}$ (Figure 1C). Furthermore, lanes 1, 2, 3, 4, 5, 6, 7, 9, and 10 were similar in that they showed the absence of a band with a size smaller than $1 \mathrm{~kb}$. The results suggested that the colony coded 8 (lane 8 ) may carry the recombinant pETBlue-2 with Zat12 insert at the desired orientation. This assumption was tested through isolation of the plasmid from colony coded 8 , which was followed by sequencing. The results agreed with the gene map published in the pETBlue-2 Cloning Kits User Manual. Hence, the results suggested that Zat12 was inserted into the cloning site of pETBlue-2 at the desired orientation. The converted sequence of ZAT12 showed the ZAT12 sequence-tagged $6 x$ His had a band with a size of $1 \mathrm{~kb}$ (refer to Figure 3). The results further confirmed that these plasmid DNA samples were $\mathrm{pENTR}^{\mathrm{TM}} / \mathrm{D}-\mathrm{TOPO}{ }^{\circledR}$ with Zat 12 inserted in the cloning site at the desired orientation. A high expression level of the ZAT12 protein was indicated by SDS-PAGE and Immunoblot. Using His antibody and ZAT12 antiserum in Immuno Blot to determine the 
specificity of ZAT12 showed that ZAT12 was expressed successfully in E. coli.

\section{Conclusions}

Recombinant ZAT12 was expressed successfully in E.coli. This study helped accumulate enough ZAT12 recombinant protein for immunizing/injecting into mice in the following study of ZAT12 antibody generation.

\section{Acknowledgements}

The author would like to convey his her deep thanks to the Laboratory of Botany, Faculty of Biosciences, University, Saarbrueken, Germany for their kind hospitality and support in conducting this study. A special thanks to Professor Dr. Uli Mueller, Department of Zoology, Saarland University, for his kind help in producing anti-ZAT12 antiserum.

\section{References}

Ben Daniel B. H., Cattan E., Wachtel C., Avrahami D., Glick Y., Malichy A., Gerber D. \& Miller G. (2016). Plant Identification of novel transcriptional regulators of Zat12 using comprehensive yeast one-hybrid screens. Physiologia Plantarum. 157(4): 422-441.

Cheong Y. H., Chang H. S., Gupta R., Wang X., Zhu T. \& Luan S. (2002). Transcriptional profiling reveals novel interactions between wounding, pathogen, abiotic stress, and hormonal responses in Arabidopsis. Plant Physiology. 129(2): 661-677.

Ciftci-Yilmaz S. \& Mittler R. (2008). The zinc finger network of plants. Cellular and Molecular Life Sciences. 65: 1150-1160.

Davletova S., Schlauch K., Coutu J. \& Mittler R. (2005). The zinc-finger protein Zat12 plays a central role in reactive oxygen and abiotic stress signaling in Arabidopsis. Plant Physiology. 139(2): 847-856.

De Abreu Neto J. B. \& Frei M. (2015). Microarray metaanalysis focused on the response of genes involved in Redox Homeostasis to Diverse Abiotic stresses in rice. Frontiers in Plant Science. 6: 1260.

Englbrecht C. C., Schoof H. \& Böhm S. (2004). Conservation, diversification and expansion of $\mathrm{C} 2 \mathrm{H} 2$ zinc finger proteins in the Arabidopsis thaliana genome. BMC Genomics. 5(1): 39.

Fowler S. \& Thomashow M. F. (2002). Arabidopsis transcriptome profiling indicates that multiple regulatory pathways are activated during cold acclimation in addition to the CBF cold response pathway. The Plant Cell. 14(8): 1675-1690.
Gamsjaeger R., Liew C. K., Loughlin F. E., Crossley M. \& Mackay J. P. (2007). Sticky fingers: zinc-fingers as protein-recognition motifs. Trends in Biochemical. Sciences. 32(2): 63-70.

Iida A., Kazuoka T., Torikai S., Kikuchi H. \& Oeda K. (2000). A zinc finger protein RHL41 mediates the light acclimatization response in Arabidopsis. The Plant Journal. 24(2): 191-203.

Kagale S., Links M. G. \& Rozwadowski K. (2010). Genome-wide analysis of ethylene-responsive element binding factor-associated amphiphilic repression motif-containing transcriptional regulators in Arabidopsis. Plant Physiology. 152(3): 1109-1134.

Kiełbowicz-Matuk A. (2012). Involvement of plant $\mathrm{C}(2) \mathrm{H}(2)$-type zinc finger transcription factors in stress responses. Plant Science. 185-186: 78-85.

Kreps J. A., Wu Y., Chang H. S., Zhu T., Wang X. \& Harper J. F. (2002). Transcriptome changes for Arabidopsis in response to salt, osmotic, and cold stress. Plant Physiology. 130(4): 2129-2141.

Laemmli U. K. (1970). Cleavage of structural proteins during assembly of head of bacteriophage T4. Nature. 227: 680-685.

Le C. T. T., Brumbarova T., Ivanov R., Stoof C., Weber E., Mohrbacher J., Fink-Straube C. \& Bauer P. (2016). Zinc finger of Arabidopsis thaliana 12 (ZAT12) interacts with FER-LIKE IRON DEFICIENCYINDUCED TRANSCRIPTION FACTOR (FIT) linking iron deficiency and oxidative stress responses. Plant Physiology. 170(1): 540-557.

Miller G., Shulaev V. \& Mittler R. (2008). Reactive oxygen signaling and abiotic stress. Physiologia Plantarum. 133(3): 481-489.

Meissner R. \& Michael A. J. (1997). Isolation and characterisation of a diverse family of Arabidopsis two and three-fingered $\mathrm{C} 2 \mathrm{H} 2$ zinc finger protein genes and cDNAs. Plant Molecular Biology. 33(4): 615-624.

Mittler R., Kim Y., Song L., Coutu J., Coutu A., CiftciYilmaz S., Lee H., Stevenson B. \& Zhu J. K. (2006). Gain- and loss-of-function mutations in Zat10 enhance the tolerance of plants to abiotic stress. FEBS Letters. 580(28-29): 6537-6542.

Peng J., Li Z., Wen X., Li W., Shi H., Yang L., Zhu H. \& Guo H. (2014). Salt induced stabilization of EIN3/EIL1 confers salinity tolerance by deterring ROS accumulation in Arabidopsis. PLoS Genetics. 10(10): e1004664.

Rizhsky L., Davletova S., Liang H. \& Mittler R. (2004). The zinc finger protein Zat12 is required for cytosolic ascorbate peroxidase 1 expression during oxidative stress in Arabidopsis. Journal of Biological Chemistry. 279(12): 11736-11743.

Rychlik W., Spencer W. J. \& Rhoads R. E. (1990). Optimization of the annealing temperature for amplification in vitro. Nucleic Acids Research. 18(21): 6409-6412. 
Sambrook J. \& Rusell D. W. (2001). Molecular Cloning: A laboratory manual. Cold Spring Harbor Laboratory Press. 1(2-3):1-2344.

Vanderauwera S., Zimmermann P., Rombauts S., Vandenabeele S., Langebartels C., Gruissem W., Inzé D. \& Van Breusegem F. (2005). Genome-wide analysis of hydrogen peroxide-regulated gene expression in Arabidopsis reveals a high light-induced transcriptional cluster involved in anthocyanin biosynthesis. Plant Physiology. 139(2): 806-821.

Vogel J. T., Zarka D. G., Van Buskirk H. A., Fowler S. G. \& Thomashow M. F. (2005). Roles of the CBF2 and ZAT12 transcription factors in configuring the low temperature transcriptome of Arabidopsis. The Plant Journal. 41(2): 195-211. 